\title{
TOPOLOGIA UNIFICADA DOS ORBITAIS MOLECULARES DE FRONTEIRA PARA EXPLICAR REAÇÕES PERICÍCLICAS
}

\author{
Silvio Cunha** \\ Instituto de Química, Universidade Federal da Bahia, Campus de Ondina, 40170-115 Salvador, BA, Brasil.
}

Recebido em 18/10/2017; aceito em 04/01/2018; publicado na web em 08/02/2018

\begin{abstract}
UNIFIED TOPOLOGY OF FRONTIER MOLECULAR ORBITALS TO EXPLAIN PERICYCLIC REACTIONS. In this work is proposed a didactic alternative vision to teach pericyclic reactions. To this end, they were classified according to the connectivity of reactants systems, as connected atom reactions (CAR) and the other possibility, as not connected atom reactions (NOCAR). Electrocyclic reactions and sigmatropic rearrangements are examples of CAR categorization, and the cycloadditions being NOCAR. With these two categories, a pedagogical alternative description is proposed to the orbital topology in reactants to explain the pericyclic reactions. According to this classification, the HOMO's topology is adequate to all CAR, while the traditional HOMO-LUMO's topology is adequate to NOCAR. All issues typically deduced by the traditional treatment, such as allowed and forbidden reactions, and stereochemical aspects are also explained by the alternative approach.
\end{abstract}

Keywords: orbital topology; sigmatropic reactions; organic chemistry teach

\section{INTRODUÇÃO}

As reações pericíclicas estão entre os temas da Química Orgânica que os estudantes de graduação e pós-graduação têm dificuldade de aprendizado; nem tanto as eletrociclizações e as cicloadições, como a reação de Diels-Alder, mas principalmente os rearranjos sigmatrópicos como os de Cope, Claisen e Wittig, entre outros. A beleza desse conjunto de reações e seu valor histórico, sem falar nas aplicações, ficam assim comprometidos.

Por se tratar de reações cujos mecanismos não apresentam os efeitos típicos de solventes nem os intermediários clássicos das reações radicalares e iônicas, temas bem trabalhados ao longo dos anos iniciais da formação, ${ }^{2}$ pode residir nestes fatos a origem da dificuldade apresentada. Todavia, isto pode ser apenas uma das faces da origem do problema, pois o modo como as reações pericíclicas são explicadas através das representações dos orbitais moleculares envolvidos pode ser a outra.

Um aspecto didático que torna o aprendizado de Química Orgânica mais facilitado é a representação pictórica dos seus fenômenos. A explicação fica facilitada se uma lógica comum emana entre argumentação e representação. Argumentações similares, explícitas ou subjacentes, sobre modelos teóricos devem conduzir a conclusões de validade também similares. Quando o ensino de química orgânica envolvendo orbitais moleculares ocorre com esta premissa, a apreensão do conhecimento assim trabalhado é mais eficaz para o estudante. ${ }^{3}$ Todavia, nos textos didáticos há alternância de argumentação na explicação do conjunto das reações pericíclicas, quando se passa de um tipo de reação para outro, Figura $1 .^{4}$

Para exemplificar a alternância mencionada, na Figura 1 são comparadas as descrições das reações de cicloadição, de eletrociclização e rearranjos sigmatrópicos. Assim, na reação de Diels-Alder que, quanto à conectividade ocorre entre átomos reativos contidos em dois conjuntos não diretamente interligados (não contínuos), a topologia do par de orbitais HOMO-LUMO é empregada para explicar a reação, enquanto que na eletrociclização, que ocorre em

*e-mail: silviodc@ufba.br

"Programa de Pós-Graduação em Ciências, Energia e Ambiente, Centro Interdisciplinar em Energia e Ambiente, Universidade Federal da Bahia

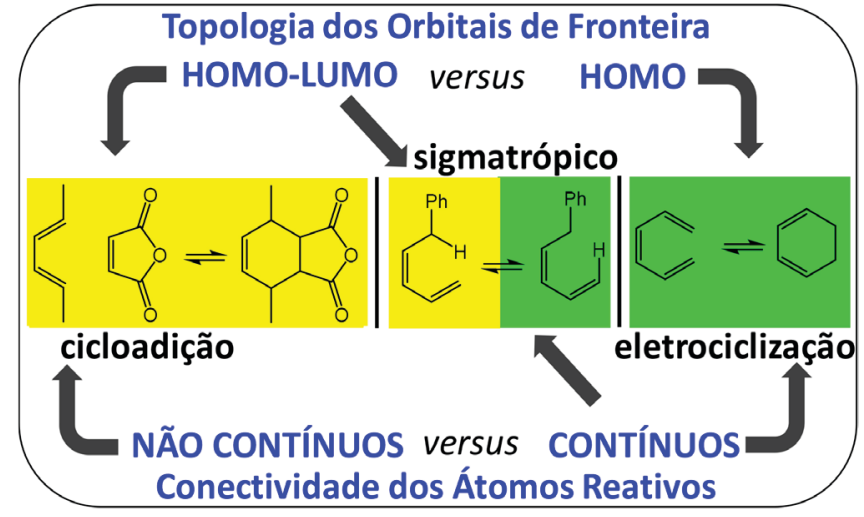

Figura 1. Alternância de argumentação, similaridades e diferenças entre reações pericíclicas térmicas

um conjunto de átomos reativos contínuos, a topologia do HOMO é suficiente para explicar a transformação, Figura $1 .{ }^{4}$ Contudo, na migração [1,5] de hidrogênio, que ocorre envolvendo um conjunto de átomos interligados e contínuos, de modo similar à eletrociclização, a racionalização é feita de forma análoga à reação de Diels-Alder, empregado o par HOMO-LUMO (da ligação sigma C-H e o do sistema conjugado, respectivamente). A necessidade desta alternância é explicada, nos textos didáticos, para preservar a observância ao princípio da conservação da simetria dos orbitais moleculares numa reação química. ${ }^{4,5}$

O emprego exclusivo do HOMO nas eletrociclizações ou do par HOMO-LUMO nas cicloadições e nos rearranjos sigmatrópicos (e nas respectivas reações reversas) pode parecer evidente para que se tenha a adequada combinação da simetria dos orbitais moleculares, mas, como veremos, não é nos rearranjos sigmatrópicos, e os textos didáticos não explicitam as razões pelas quais estes rearranjos são sempre racionalizados empregando o par dos orbitais HOMO-LUMO.

Apresentamos aqui, como descrição alternativa, propostas de categorização das reações pericíclicas e de unificação da topologia dos orbitais moleculares de fronteira para as explicar, com ênfase nos rearranjos sigmatrópicos. ${ }^{7}$ 
RAC e RANC: categorias das reações pericíclicas e topologia dos orbitais moleculares de fronteira

Das reações pericíclicas, as eletrociclizações e os rearranjos sigmatrópicos são exclusivamente intramoleculares, pois as cicloadições e as reações queletrópicas podem ser tanto intra quanto intermoleculares. ${ }^{4}$ Todavia, a análise do conjunto faz emanar duas outras categorias que estão relacionadas com a conectividade dos átomos que reagem: as reações que ocorrem entre átomos contínuos (RAC) de uma única molécula, como as eletrociclizações e todos os rearranjos sigmatrópicos; e as reações que ocorrem entre átomos não-contínuos (RANC) de uma única molécula ou de duas moléculas diferentes, como as cicloadições e as reações queletrópicas.

Esta categorização das reações pericíclicas tem apenas função pedagógica e pode contribuir com uma abordagem mais didática pois, em função dela, podemos unificar as topologias dos orbitais moleculares para cada tipo de reação. Assim, duas reações aparentemente não conexas podem ser visualizadas como do mesmo tipo, RAC ou RANC, e então emana uma lógica comum de mesma topologia dos orbitais para explicar reações de igual categoria, como ilustrado na Figura 2.

$\mathrm{Na}$ primeira categoria, a reação de átomos contínuos (RAC) ocorre envolvendo um sistema contínuo de elétrons $\pi$ reativos, contidos num segmento de átomos também contínuos de uma única molécula (reação intramolecular), e são exemplos as eletrociclizações e os rearranjos sigmatrópicos [1,3], [1,5] e [1,7], bem como os rearranjos sigmatrópicos $[3,3]$ e [2,3]. Na segunda categoria, a reação de átomos não-contínuos (RANC) ocorre com a participação de dois sistemas não-contínuos de elétrons $\pi$ reativos contidos em dois segmentos de átomos de duas moléculas diferentes (reação intermolecular), ou contidos em duas porções diferentes de uma mesma molécula, mas separados por espaçador de átomos com sistema de elétrons não reativos, portanto em dois segmentos de átomos não-contínuos da molécula (reação intramolecular), e são exemplos as cicloadições.

Na identificação do sistema de elétrons $\pi$ reativos são levados em consideração tanto os elétrons de ligações formalmente $\pi$, no caso das eletrociclizações e cicloadições, quanto os elétrons de ligações formalmente $\pi$ e de uma ligação sigma adjacente à $\pi$, no caso dos rearranjos sigmatrópicos. Para exemplificar, na Figura 2 estão ilustradas as analogias entre o 1,3-butadieno e o propeno (Quadro 1) e entre o 1,3-butadieno e o 1-buteno (Quadro 4), que são protótipos para explicar a reação de eletrociclização e os rearranjos sigmatrópicos 1,3 de hidrogênio e de grupo alquila, respectivamente.

O cômputo dos quatro elétrons $\pi$ do sistema reativo no 1,3-butadieno demanda dois orbitais moleculares, sendo assim $\Psi_{2}$ o seu orbital HOMO. Como em polienos conjugados o número de planos nodais de um dado orbital molecular $\Psi_{\mathrm{n}}$ é n-1, o $\mathrm{HOMO} \Psi_{2}$ do 1,3-butadieno possui um plano nodal localizado no centro geométrico das ligações $\pi$ conjugadas, como indicado na Figura 2 (itens a e $\underline{b}$ do Quadro 1).

Aplicando a analogia estrutural entre o 1,3-butadieno e o propeno indicada na Figura 2 (item a do Quadro 1), a ligação sigma $\mathrm{C}_{3}-\mathrm{H}_{4}$ do propeno passa a contribuir com o sistema reativo e seus dois elétrons são computados como equivalentes a um par de elétrons $\pi$. Assim, o plano nodal é localizado entre os mesmos átomos nestas

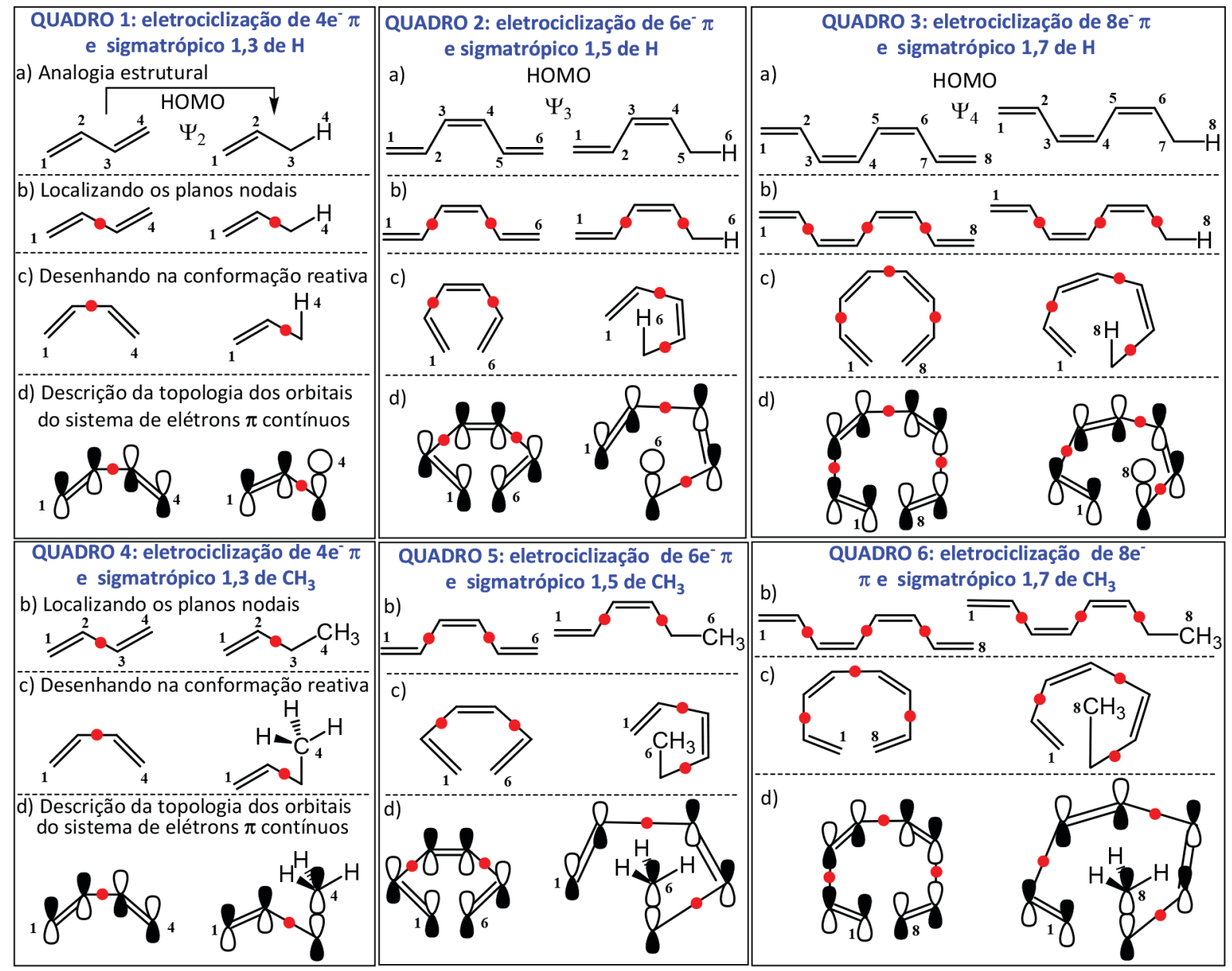

Figura 2. Dedução da topologia dos orbitais HOMO para as reações térmicas do tipo RAC: analogia entre as topologias de eletrociclizações e rearranjos sigmatrópicos [1,3], [1,5] e [1,7] 
duas moléculas, ou seja, os átomos 2 e 3 de cada um dos sistemas de elétrons reativos (item $\underline{\mathrm{b}}$ do Quadro 1).

Uma vez determinado a posição do plano nodal, a conformação reativa de cada molécula é escrita aproximando-se os átomos do sistema de elétrons $\pi$ reativos (item $\underline{c}$ do Quadro 1 ). Para o 1,3-butadieno os átomos 1 e 4 ficam coplanares, mas no propeno a ligação sigma $\mathrm{C}_{3}-\mathrm{H}_{4}$ fica perpendicular ao plano formado pelos átomos 1,2 e 3 . Dessa forma, ao inserir os orbitais que descrevem o HOMO das duas moléculas, a analogia permite deduzir a topologia na orientação adequada para julgar se e como ocorre cada uma das reações pericíclicas.

Aplicando sucessivamente o mesmo procedimento para as reações térmicas do tipo RAC com número crescente de ligações $\pi$, podemos deduzir a topologia dos orbitais moleculares de fronteira HOMO de forma análoga entre as eletrociclizações e os rearranjos sigmatrópicos $[1,5]$ e $[1,7]$ como indicado, respectivamente, nos Quadros 2 e 3 da Figura 2 onde, nos polienos conjugados, os planos nodais do orbital molecular HOMO são localizados equidistantes do centro geométrico das ligações $\pi$ conjugadas.

Da mesma maneira, os rearranjos sigmatrópicos [1,3], [1,5] e $[1,7]$ de grupo alquila têm a topologia dos HOMO deduzida apenas substituindo o $\mathrm{H}$ da ligação sigma $\mathrm{C}$-H por um grupo alquila, ilustrado por um grupo $\mathrm{CH}_{3}$ nos Quadros 4 a 6 da Figura 2.

Os rearranjos sigmatrópicos ocorrem com a quebra de uma ligação sigma em uma das extremidades do sistema de átomos contínuos concomitante à formação de uma nova ligação sigma na outra extremidade do mesmo sistema. Quando as duas ligações envolvidas nesta reação são localizadas na mesma face do sistema de átomos contínuos, a migração é denominada suprafacial. Quando uma ligação é rompida em uma das faces do sistema de átomos contínuos e a outra ligação é formada na face oposta, a migração é denominada antarafacial. ${ }^{4}$ Nas Figuras 3 e 4 são empregadas linhas curvas tracejadas para exemplificar estes dois modos de reação. ${ }^{8}$

De posse das topologias deduzidas para o HOMO de cada molécula é possível aplicar as regras de simetria dos orbitais para julgar se o rearranjo sigmatrópico em análise é permitido ou proibido, supra ou antarafacial, com ou sem inversão de configuração nos casos da migração do grupo alquila. Também é possível explicar as questões estereoquímicas. Para fins de comparação das reações do tipo RAC comentadas na Figura 2, as racionalizações destas reações pericíclicas são apresentadas nas Figuras 3 e 4, e as topologias alternativa e convencional são comparadas.

Nas cicloadições e eletrociclizações a representação das topologias dos orbitais é a convencional, encontrada nos textos didáticos, com a descrição das moléculas no estado fundamental, mas para os rearranjos sigmatrópicos a análise é efetuada com a topologia aqui deduzida e comparada com a convencional. A abordagem alternativa levar às mesmas conclusões do tratamento convencional, com a vantagem de haver simplificação de dedução da topologia, pois o HOMO de cada molécula é sempre empregado para todas as reações do tipo RAC (eletrociclizações e rearranjos sigmatrópicos).

A análise da Figura 3 revela que o emprego do HOMO para a migração [1,3] de hidrogênio indica que a migração é proibida suprafacialmente pois os orbitais que estão na mesma face do sistema de átomos contínuos estão fora de fase (sinais contrários indicados por orbital preenchido com cor interagindo com orbital sem cor). Apesar dos sinais em fase nos átomos $\mathrm{C} 1$ e $\mathrm{H} 4$ indicarem que a migração antarafacial é teoricamente possível, o tamanho pequeno da cadeia carbônica não permite que ocorra assim, pois não há possibilidade de migração envolvendo o mesmo átomo de hidrogênio $\mathrm{H} 4$ em que uma ligação C3-H4 esteja sendo rompida pela face de cima e outra $\mathrm{C} 1-\mathrm{H} 4$ sendo simultaneamente formada pela face de baixo, o que denominamos como sendo proibido por geometria.

Para a migração $[1,3]$ de carbono (grupo alquila) o HOMO do sistema de 4 elétrons $\pi$ explica a migração suprafacial com inversão de configuração, da mesma forma que a abordagem tradicional, mas sem a necessidade de arbitrariamente fixar os dois fragmentos HOMO e LUMO dentro da uma única molécula, Figura 3. Note que esta inversão de configuração no modo suprafacial é similar ao que ocorre numa $\mathrm{S}_{\mathrm{N}} 2$ pois os sinais de mesma fase nos orbitais dos carbonos $1 \mathrm{e}$ 4, indicados na Figura 3 por uma linha tracejada, só podem interagir construtivamente se esta nova ligação sigma $\mathrm{C} 1-\mathrm{C} 4$ for formada pelo

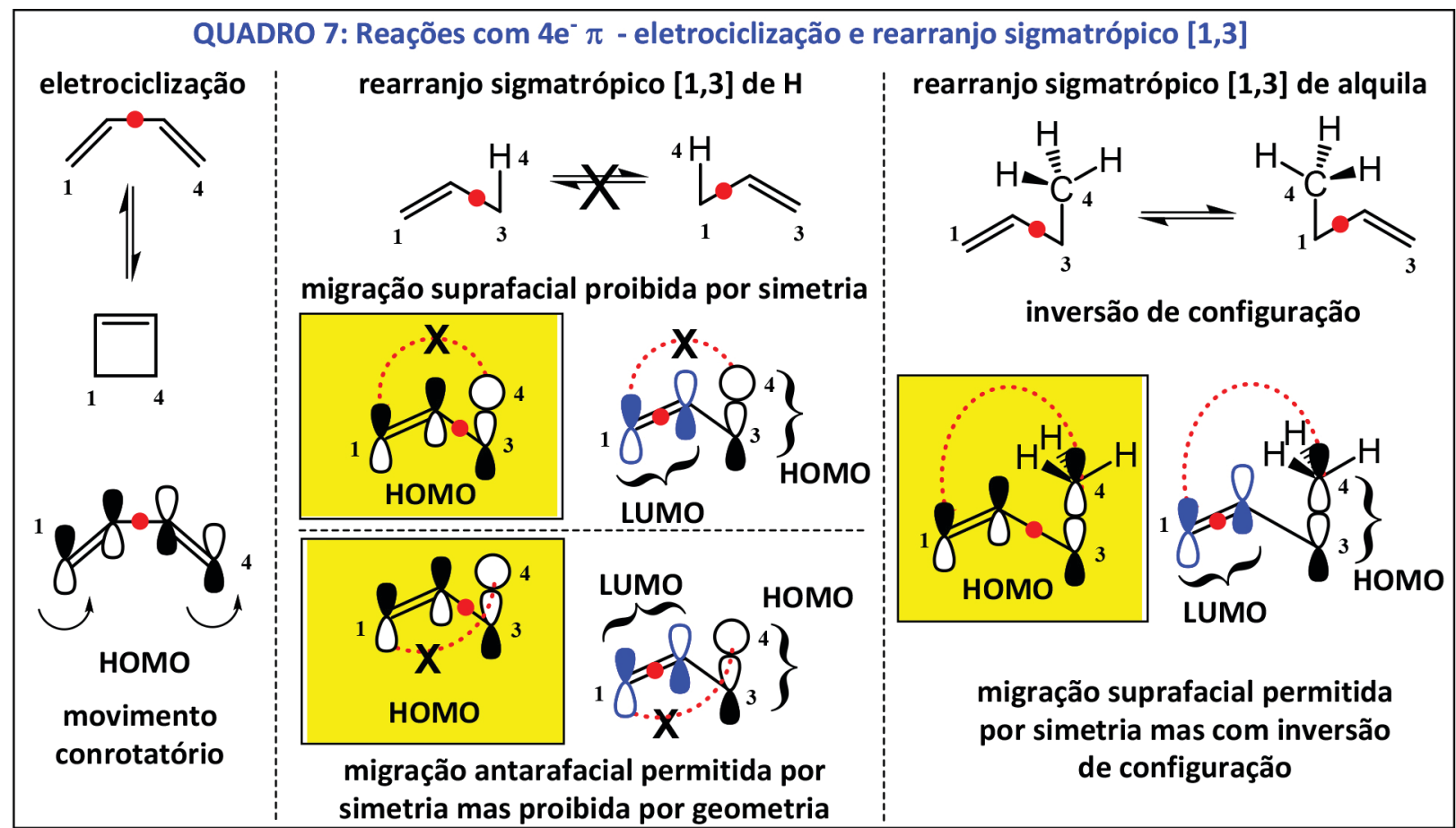

Figura 3. Comparação das topologias alternativa (em destaque) e convencional para as reações térmicas do tipo RAC: eletrociclização e rearranjo sigmatrópico [1,3] 
lado oposto à ligação sigma C3-C4 que se rompe entre os átomos $4 \mathrm{e}$ 3. Pelo mesmo motivo da migração $[1,3]$ de hidrogênio, a migração antarafacial [1,3] de carbono é proibida por geometria.

Na mesma linha de raciocínio, empregando o HOMO destacado nos Quadros da Figura 4 se deduz que, de acordo com as fases dos orbitais das extremidades do sistema de átomo reativos contínuos, a migração [1,5] de hidrogênio é permitida suprafacialmente, e que a migração [1,5] de carbono é também permitida suprafacialmente com retenção de configuração pois as fases de mesmo sinal, indicada pela linha tracejada no Quadro 8 da Figura 4, podem desenvolver a interação ligante entre os átomos C1-C6 pelo mesmo lado da ligação C5-C6 que se rompe. A interação das fases entre os átomos C1-C6 de modo antarafacial, apesar de teoricamente permitida, desenvolve um estado de transição muito tensionado quando comparado ao modo suprafacial, em função do tamanho pequeno de átomos da cadeia carbônica, comprometendo o paralelismo do sistema de elétrons $\pi$ reativos, e faz com que a migração antarafacial com inversão de configuração não prevaleça.

Por sua vez, na migração [1,7] de hidrogênio, a análise dos orbitais dos átomos que interagem na extremidade do sistema de átomos contínuos revela que a migração do $\mathrm{H} 8$ para o $\mathrm{C} 1$ só é permitida antarafacialmente. Neste caso, a migração ocorre porque o tamanho da cadeia contínua de átomos do sistema reativo proporciona a aproximação do $\mathrm{C} 1$ ao $\mathrm{H} 8$ e a sobreposição das fases de mesmo sinal localizadas em lados opostos do sistema $\pi$ reativo, como indicado com a linha tracejada no Quadro 9 da Figura 4, com avanço da reação com um grau de torção da cadeia carbônica que não compromete significativamente o necessário paralelismo do sistema de elétrons $\pi$ reativos. Para a correspondente migração $[1,7]$ de carbono, a reação é permitida suprafacialmente com inversão de configuração no $\mathrm{C} 8$,

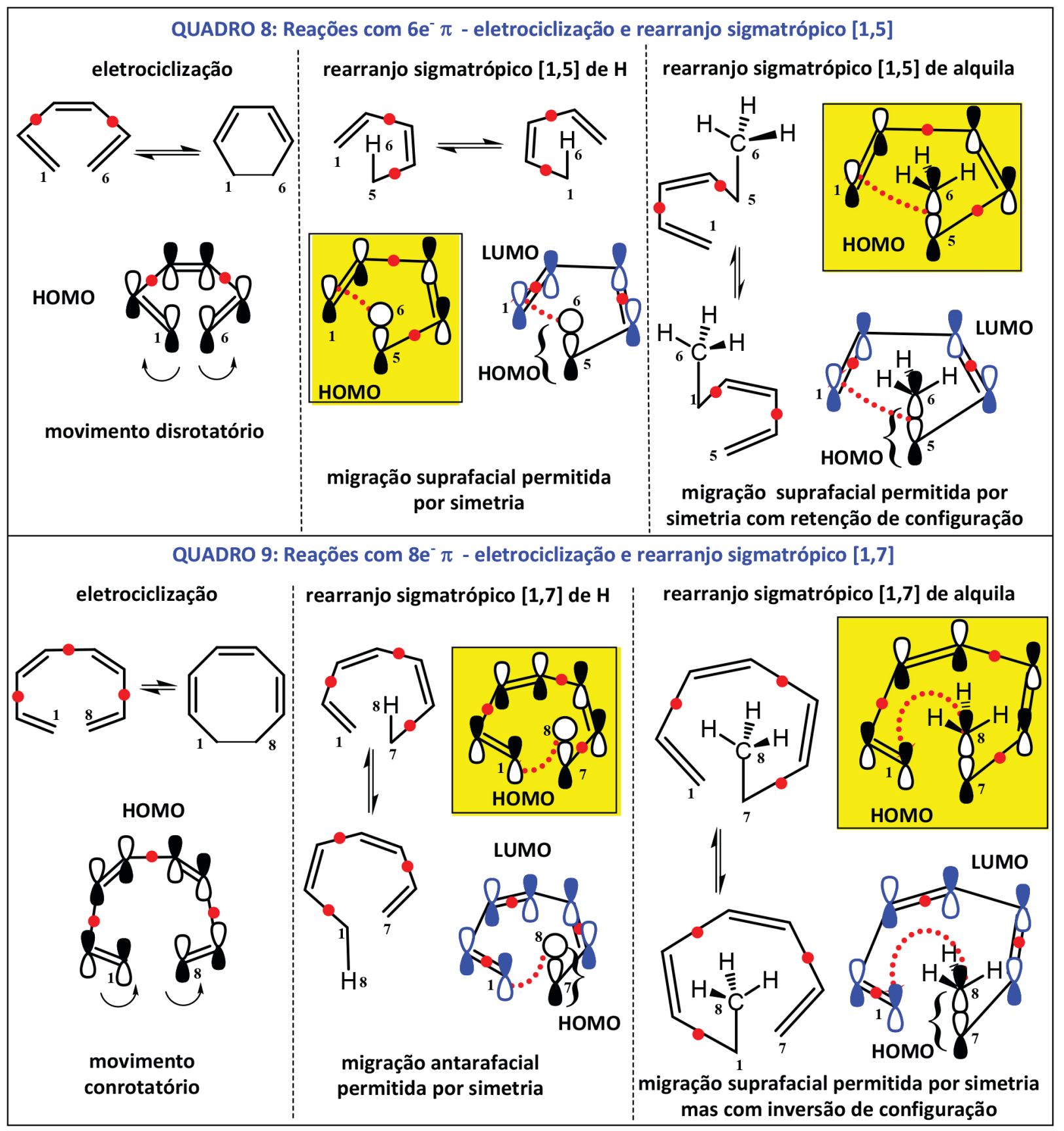

Figura 4. Comparação das topologias alternativa (em destaque) e convencional para as reações térmicas do tipo RAC: eletrociclizações e rearranjos sigmatrópicos [1,5] e [1,7] 
pelos mesmos motivos discutidos para a migração [1,5] de carbono. Todavia, esta mesma migração de forma antarafacial com retenção de configuração no C8 é desfavorecida pois, para ocorrer, requer a aproximação do $\mathrm{C} 8$ ao $\mathrm{C} 1$ por faces opostas, desenvolvendo uma torção acentuada da cadeia carbônica que compromete o paralelismo do sistema de elétrons $\pi$ reativos de forma muito superior ao que ocorre na migração antarafacial de hidrogênio.

No Esquema 1 são apresentados exemplos dos rearranjos sigmatrópicos de hidrogênio e de grupo alquila, onde é feita a racionalização de cada reação empregando a topologia alternativa aqui desenvolvida. ${ }^{9-13}$

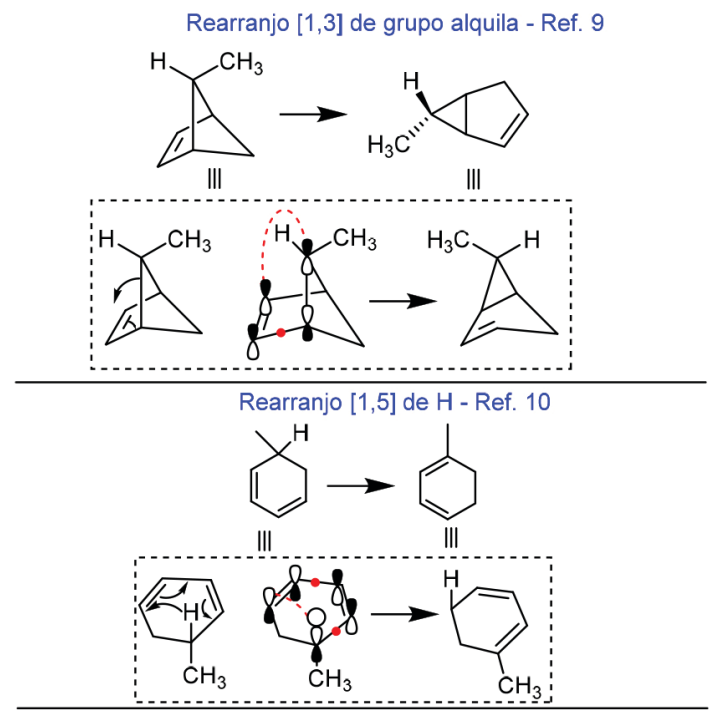

Rearranjo [1,5] de grupo alquila - Ref. 11
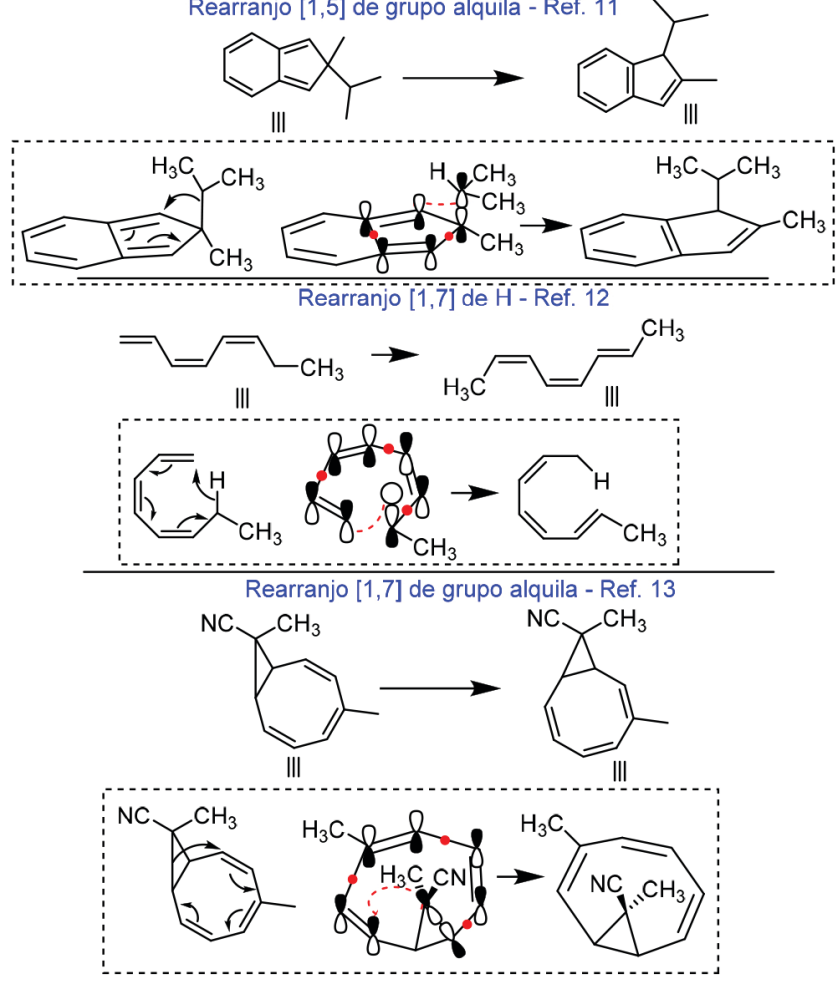

Esquema 1. Exemplos de rearranjos sigmatrópicos [1,3], [1,5] e [1,7] racionalizados com a topologia alternativa

Do exposto, as reações pericíclicas térmicas do tipo RAC podem ser explicadas sempre empregando o HOMO do sistema contínuo de elétrons $\pi$ reativos da molécula. Para verificar a validade dessa afirmação, os rearranjos sigmatrópicos [3,3] e [2,3] foram analisados com o procedimento de dedução da topologia dos orbitais HOMO, como descrito na Figura 5.

Mesmo nos livros-texto avançados de química orgânica não é comum encontrar a descrição da topologia dos orbitais referentes aos rearranjos sigmatrópicos para além os rearranjos [1,3], [1,5] e $[1,7] .{ }^{4}$ Por este motivo, apresentamos a comparação entre o tratamento convencional e o alternativo aqui proposto para os rearranjos sigmatrópicos [3,3] e [2,3]. O tratamento convencional da topologia dos orbitais explica estas duas reações intramoleculares considerando, simultaneamente na mesma molécula, uma porção HOMO e outra LUMO conectadas por uma ligação sigma envolvida na reação. Já na topologia alternativa, de forma mais simples, é empregado apenas o orbital HOMO de 6 elétrons $\pi$ envolvendo os átomos 1 a 6 e 1 a 5 nos rearranjos [3,3] e [2,3], respectivamente, Figura 5.

Como protótipo para explicar o rearranjo sigmatrópico [3,3] é empregada, na Figura 5, a analogia entre o 1,3,5-hexatrieno e o 1,5-hexadieno, seguindo o procedimento comentado na Figura 2. Como em polienos conjugados o número de planos nodais de um dado orbital molecular $\Psi_{\mathrm{n}}$ é n-1, no 1,3,5-hexatrieno temos três pares de elétrons $\pi$ que demandam três orbitais moleculares, sendo assim $\Psi_{3}$ o HOMO. Portanto, dois planos nodais são localizados equidistantes do centro geométrico das ligações $\pi$ conjugadas, como indicado no Quadro 10 da Figura 5. É possível redesenhar o 1,5-hexadieno com os carbonos 1 e 6 se aproximando para permitir a sobreposição em fase dos orbitais localizados nestes dois átomos, mantendo a disposição espacial dos demais átomos na forma cadeira. De forma análoga, o rearranjo sigmatrópico [2,3] é explicado com a topologia do HOMO deduzida, tomando como analogia o ânion pentadienila, como indicado no Quadro 11 da Figura 5.

No Esquema 2 são apresentados exemplos dos rearranjos [3,3] e $[2,3]$ onde é feita a racionalização de cada reação empregando a topologia alternativa aqui desenvolvida. Para o último, são exemplificados casos onde o intermediário é neutro ou dipolar. ${ }^{14-16}$

Uma vez analisadas as reações pericíclicas do tipo RAC, resta a interpretação das reações do tipo RANC. Na Figura 6 duas reações RANC são comparadas, a reação Ene e a Diels-Alder, onde o par HOMO-LUMO é sempre necessário para a racionalização das reações desta categoria. Note que na reação Ene, mesmo sendo descrita a topologia convencional, no fragmento do propeno é empregado o HOMO como deduzido na Figura 2. Adicionalmente, como as ligações sigma envolvidas na reação Ene (uma sigma se forma e outra é rompida) e na Diels-Alder (duas ligações sigma são formadas) estão localizadas na mesma face de cada extremidades dos sistemas de átomos contínuos de cada molécula, em cada reação, o processo é denominado suprafacial, Figura 6. No Esquema 3 são apresentados exemplos de reação Ene e Diels Alder. ${ }^{17,18}$

\section{CONCLUSÃO}

As reações pericíclicas térmicas do tipo RAC podem ser explicadas sempre empregando a topologia do HOMO do sistema contínuo de elétrons $\pi$ reativos da molécula, e as do tipo RANC são explicadas sempre empregando a topologia do par HOMO-LUMO dos sistemas de elétrons $\pi$ reativos da molécula ou das moléculas reagentes, e a Figura 7 sumaria o cenário alternativo das reações pericíclicas. $^{8}$

A proposta alternativa elaborada é explicação operacional para fins de ensino, intencionando ser uma abordagem algo mais simples que a tradicionalmente apresentada nos textos didáticos, pois a sugestão das duas categorias de reações RAC ou RANC proporciona a analogia da topologia dos orbitais entre reações do mesmo tipo. 

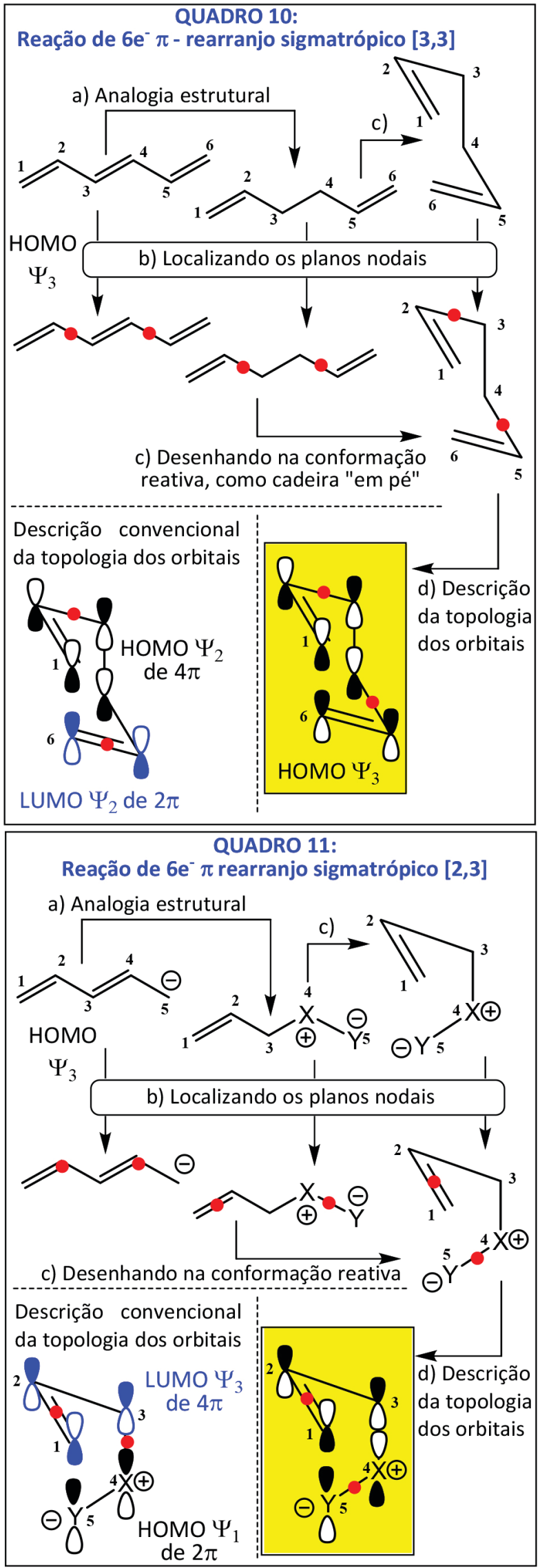

Figura 5. Dedução da topologia alternativa (em destaque) e comparação com a convencional dos orbitais moleculares de fronteira para as reações térmicas do tipo RAC: rearranjos sigmatrópicos [3,3] e [2,3]

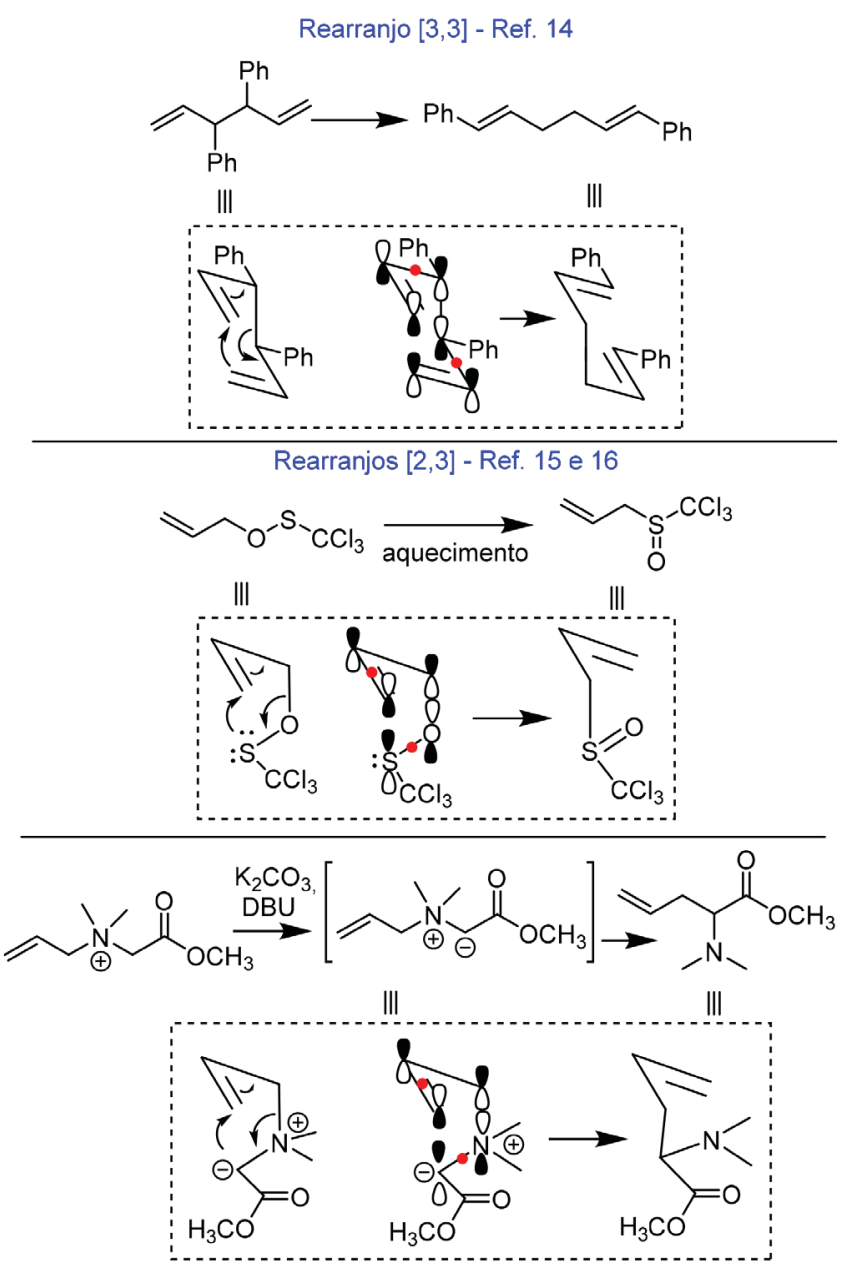

Esquema 2. Exemplos de rearranjos sigmatrópicos [3,3] e [2,3] racionalizados com a topologia alternativa

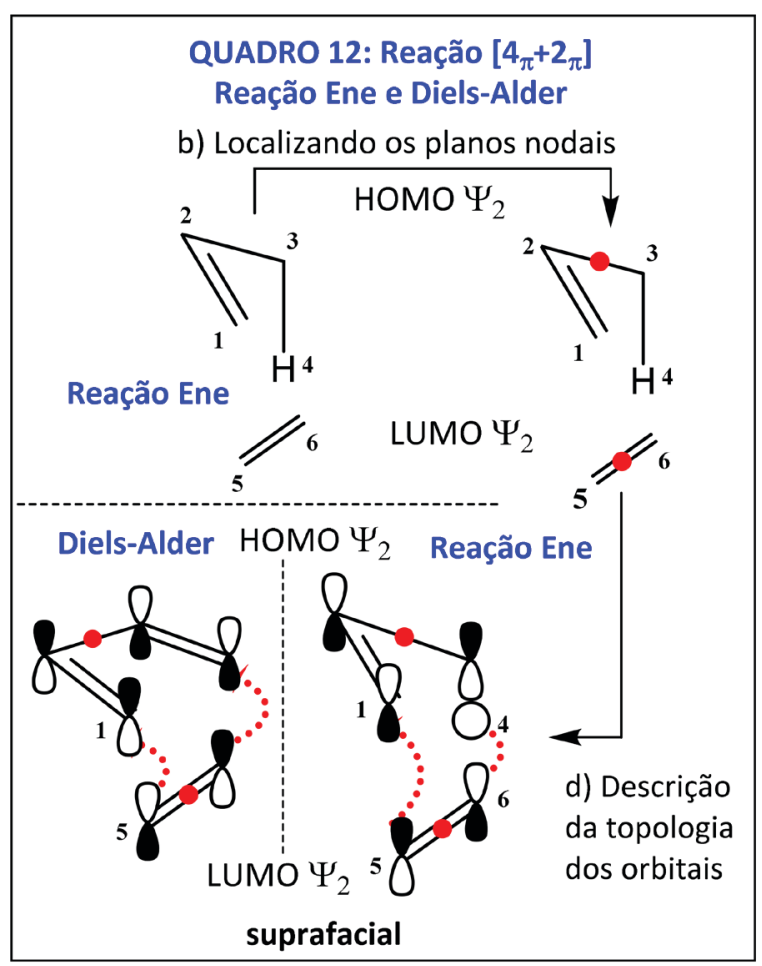

Figura 6. Dedução da topologia dos orbitais moleculares de fronteira para as reações térmicas do tipo RANC: Ene e Diels-Alder 


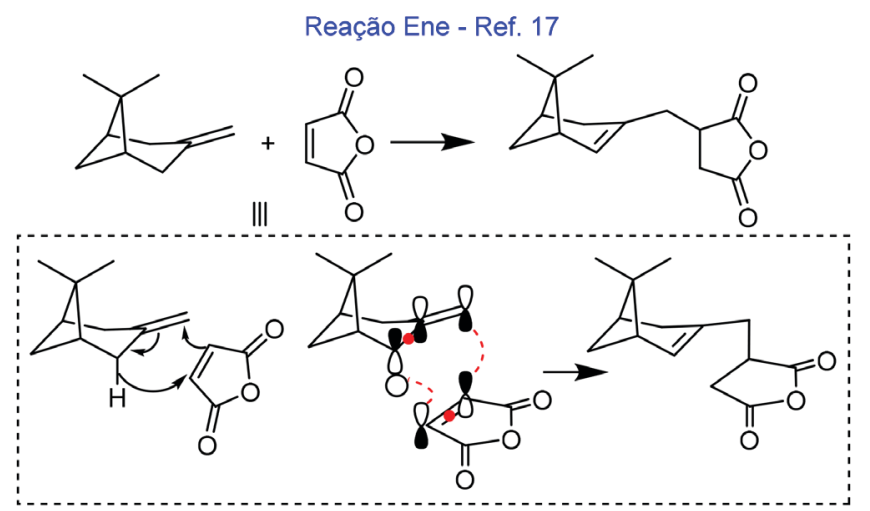

Diels-Alder - Ref. 18<smiles>C(=[P+]c1ccccc1)/[PH+]=C/[PH2+]c1ccccc1</smiles><smiles>O=C1C=CC(=O)O1</smiles><smiles>O=C1OC(=O)C2C1[C@H](c1ccccc1)C=C[C@H]2c1ccccc1</smiles>

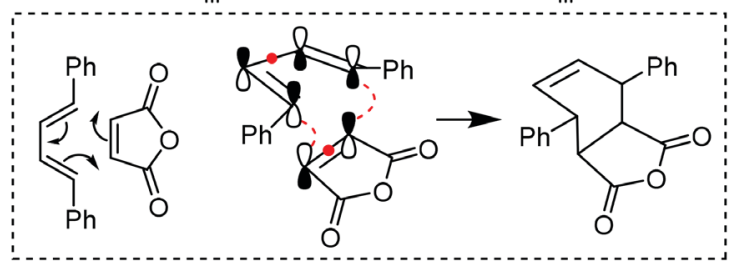

Esquema 3. Exemplos de reações Ene e Diels-Alder

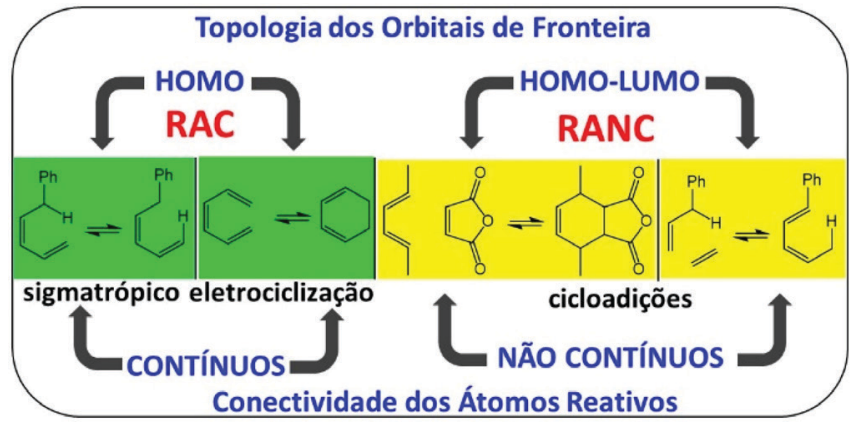

Figura 7. Panorama alternativo das reações pericíclicas térmicas

\section{AGRADECIMENTOS}

O autor agradece o suporte financeiro do Conselho Nacional de Desenvolvimento Científico e Tecnológico - CNPq, Coordenação de Aperfeiçoamento de Pessoal de Nível Superior - CAPES, e Fundação de Amparo à Pesquisa do Estado da Bahia - FAPESB, e ao CNPq a bolsa de produtividade em pesquisa e ao Instituto Nacional de Ciência e Tecnologia-INCT em Energia e Ambiente. Agradeço também aos dois Assessores anônimos que contribuiram muito com sugestões para tornar o manuscrito mais didático.

\section{REFERÊNCIAS}

1. Woodward, R. B.; Hoffmann, R.; Angew. Chem. Int. Ed. 1969, 8, 781; Woodward, R. B.; Hoffmann, R.; J. Am. Chem. Soc. 1965, 87, 395; Hoffmann, R.; Woodward, R. B.; J. Am. Chem. Soc. 1965, 87, 2046; Hoffmann, R.; Woodward, R. B.; J. Am. Chem. Soc. 1965, 87, 4388; Palazzo, T. A.; Mose, R.; Jorgensen, K. A.; Angew. Chem. Int. Ed. 2017, 56, 10033 .

2. Mullins, J. J.; J. Chem. Educ. 2008, 85, 83; Scudder, P. H.; Electron Flow in Organic Chemistry; John Wiley \& Sons: New York, 1992.

3. Martins, B. S.; Lüdtke, D. S.; Moro, A. V.; Quim. Nova 2017, 40, 342; de Freitas, J. J. R.; Avelino, R. A.; Mata, M. M. S.; Santos, C. S.; Almeida, C. L. A.; Freitas, J. C. R.; Freitas Filho, J. R.; Rev. Virtual Quim. 2017, 9, 1597; Cunha, S.; Quim. Nova 2003, 26, 948.

4. Fleming, I.; Frontier Orbitals and Organic Chemical Reactions; John Wiley \& Sons: Chichester, 1976; Fleming, I.; Molecular Orbitals and Organic Chemical Reactions; John Wiley \& Sons: Cambridge, 2009; Clayden, J., Greeves, N., Warren, S.; Organic Chemistry, $2^{\text {nd }}$ ed., Oxford University Press; 2012; Smith, M. B.; March's Advanced Organic Chemistry: Reactions, Mechanisms, and Structure, 7th ed., John Wiley \& Sons: New York, 2013; Carey, F. A.; Sundberg, R. J., Advanced Organic Chemistry, Part A: Structure and Mechanisms, 5th ed., Plenum Press: New York, 2007; Anslyn, E. V.; Dougherty, D. A., Modern Physical Organic Chemistry, University Science Books: Salsalito, 2006; Bruckner, R., Organic Mechanisms, Springer, Berlin: 2010. Merlo, A. A.; Reações Pericíclicas: uma Sinfonia de Moléculas e Elétrons, Editora da UFRGS: Porto Alegre, 2012.

5. Ranganathan, S.; Resonance 1997, 2, 41; Pearson, R. G.; J. Chem. Educ. 1981, 58, 753; Inagaki, S.; Fujimoto, H.; Fukui, K.; J. Am. Chem. Soc. 1976, 98, 4693.

6. As razões pelas quais o emprego da topologia dos orbitais moleculares dos reagentes é aplicado com sucesso na explicação das reações pericíclicas, de natureza exergônica, estão muito bem explicadas nos textos didáticos: ver a referência 4.

7. Para um debate interessante sobre rearranjo sigmatrópico [1,3], ver: Fonseca, C. S. C.; J. Chem. Educ. 2016, 93, 1781; Hare, S. R.; Tantillo, D. J.; J. Chem. Educ. 2017, 94, 988; Fonseca, C. S. C.; J. Chem. Educ. 2017, 94, 994.

8. Apesar de ilustradas apenas para as reações termicamente promovidas, as correspondentes reações pericíclicas fotoquímicas são também explicadas com a proposta alternativa aqui desenvolvida, mas empregando agora a topologia do LUMO para as reações do tipo RAC.

9. Berson, J. A.; Nelson, G. L.; J. Am. Chem. Soc. 1967, 89, 5303.

10. Marvell, E. N.; Caple, G.; Schatz, B.; Pippin, W.; Tetrahedron 1973, 29, 378.

11. Dolbier Jr., W. R.; Anapalle, K. E.; McCullagh, L. C.; Matsui, K.; Riemann, J. M.; Robison, D.; J. Org. Chem. 1979, 44, 2845.

12. Baldwin, J. E.; Reddy, V. P.; J. Am. Chem. Soc. 1987, 109, 8051.

13. Klarner, F. G.; Angew. Chem. Int. Ed. 1972, 11, 832.

14. Doering, W. von E.; Roth, W. R.; Tetrahedron 1962, 18, 67.

15. Braverman, S.; Stabinsky, Y.; Chem. Commun. 1967, 270.

16. Coldham, I.; Middleton, M. L.; Taylor, P. L.; J. Chem. Soc., Perkin Trans. 1 1997, 2951.

17. Arnold, R. T; Showell, J. S.; J. Am. Chem. Soc. 1957, 79, 419.

18. Alder, K.; Schumacher, M.; Annalen 1951, 517, 87. 